\title{
Comunidades de formigas (Hymenoptera, Formicidae) em fragmentos de Mata Atlântica situados em áreas urbanizadas
}

\author{
Maria Santina de C. Morini ${ }^{1}$, Catarina de B. Munhae ${ }^{1}$, Roberto Leung ${ }^{2}$, \\ David F. Candiani ${ }^{3}$ \& Júlio C. Voltolini ${ }^{4}$
}

1. Núcleo de Ciências Ambientais, Universidade de Mogi das Cruzes, Av. Dr. Cândido Xavier de Almeida Souza, 200, 08780-911 Mogi das Cruzes, SP. (morini@umc.br)

2. Universidade Federal do Mato Grosso, 78698-000 Pontal do Araguaia, MT.

3. Instituto Butantan, Av. Vital Brasil, 1500, 05503, São Paulo, SP.

4. Universidade de Taubaté, Departamento de Biologia, Taubaté, SP.

\begin{abstract}
Ants' communities (Hymenoptera, Formicidae) in fragments of the Atlantic Rain Forest situated in urban areas. In this paper were investigated the ants' communities that inhabit the Atlantic Rainforest fragments surrounded by an urban ecosystem well developed. The richness, the species frequency of occurrence, as well the similarity between the three areas of the city of São Paulo were investigated: Parque da Previdência (PP), Reserva Florestal "Armando Salles de Oliveira" (CUASO) and Horto Oswaldo Cruz (HOC). Pit-fall tramps were put in places where the public visitation is not allowed, during a whole week in the months of March, June, September and December of 2001. Considering the three fragments were collected 79 ant species belonging to nine subfamilies and 32 genera. The biggest species richness was presented by the Subfamily Myrmicinae and the genera Pheidole and Hypoponera. In PP were registered 62 species, in CUASO 46 and in HOC 43. The biggest similarity was found between PP and CUASO and possibly this similarity may be due to the size of both areas and to a similarity between the sites of nesting and feeding. In general, the ants' fauna is generalist, with the presence of some specialists genera as Discothyrea, Acanthognathus, Gnamptogenys, Oxyepoecus and Pyramica or genera that have feeding habits still unknown (Heteroponera and Myrmelachista). The presence of Pheidole megacephala Fabricius, 1793, Linepithema humile Mayr, 1868, Wasmannia auropunctata Roger, 1863, Paratrechina fulva Mayr, 1862, P. longicornis Latreille, 1802 and Tapinoma melanocephalum Fabricius, 1793, species characteristic of household areas, was also observed.
\end{abstract}

KEYWORDS. Atlantic Rainforest, urban ecosystem, Formicidae, richness.

RESUMO. As comunidades de formigas que vivem em fragmentos de Mata Atlântica rodeados por um ecossistema urbano bem desenvolvido, foram investigadas. Avaliou-se a riqueza, a frequiência de ocorrência das espécies bem como a similaridade entre três áreas da cidade de São Paulo: Parque da Previdência (PP), Reserva Florestal "Armando Salles de Oliveira" (CUASO) e Horto Oswaldo Cruz (HOC). Foram colocadas armadilhas do tipo "pitfall" em locais onde não ocorre visitação pública, durante uma semana, nos meses de março, junho, setembro e dezembro de 2001. Em todos os fragmentos foram coletadas 79 espécies de formigas, pertencentes a nove subfamílias e 32 gêneros. A subfamília Myrmicinae e os gêneros Pheidole e Hypoponera foram os mais ricos. No PP foram registradas 62 espécies, na CUASO 46 e no HOC 43, sendo que PP e CUASO são mais similares entre si. Tal similaridade possivelmente esteja relacionada ao tamanho de ambas as áreas e, também, a uma semelhança nos sítios de nidificação e de alimentação. No geral, a fauna de formigas é generalista, com a presença de alguns gêneros especialistas, como Discothyrea, Acanthognathus, Gnamptogenys, Oxyepoecus e Pyramica; ou de gêneros cujos hábitos alimentares ainda são desconhecidos (Heteroponera e Myrmelachista). A presença de espécies caracteristicamente de áreas domiciliares também foi constatada: Pheidole megacephala Fabricius, 1793, Linepithema humile Mayr, 1868, Wasmannia auropunctata Roger, 1863, Paratrechina fulva Mayr, 1862, P. longicornis Latreille, 1802 e Tapinoma melanocephalum Fabricius, 1793.

PALAVRAS-CHAVE. Mata Atlântica, ecossistema urbano, Formicidae, riqueza.

A maior ameaça à diversidade biológica de comunidades naturais relativamente intactas dentro e no entorno do ecossistema urbano é a destruição de habitats nativos e sua conversão a outros usos. A pavimentação desses habitats à medida que as atividades humanas se espalham destrói e fragmenta os ecossistemas remanescentes (MURPHY, 1997).

Qualquer efeito de urbanização e de atividades humanas afeta a qualidade dos habitats remanescentes ou mesmo a probabilidade do sucesso de dispersão dos animais e vegetais entre habitats residuais, quando cercados pela malha urbana (ConNor et al., 2002). Diante das novas condições impostas pela urbanização, muitas espécies não conseguem viver e as áreas com vegetação nativa nas cidades representam importantes locais de refúgio para plantas e animais não-adaptados ao ambiente urbano (RodRIGUES et al., 1993). Por outro lado, existem espécies oportunistas que se aproveitam das novas condições de colonização (MCINTYRE et al., 2001) e que podem ocasionar a extinção local das espécies nativas (Suarez et al., 1998; Crooks \& Soulé, 1999).

Dentre os insetos sociais, as formigas representam um grupo importante para estudos sobre o impacto da urbanização na estrutura e funcionamento de suas comunidades (LÓPEZ-MORENO et al., 2003). Entretanto ainda existem poucos trabalhos envolvendo espécies que habitam os ecossistemas urbanos sobre as interações entre o ser humano e as diferentes espécies, bem como sobre os efeitos da urbanização nos ecossistemas, nas comunidades, nas espécies e nas populações (LÓPEZ-MoReno \& DiAZBETANCOURT,1995; BLAIR, 1996; MCINTYRe, 2000).

Como no Brasil praticamente todos os artigos que abordam as comunidades de formigas que vivem nos ecossistemas urbanos estão relacionados aos ambientes 
domiciliar e hospitalar (Delabie et al., 1995; Bueno \& CAMPOS-FARINHA, 1999; CAMPOS-FARINHa et al., 2002), este trabalho objetivou estudar as comunidades de formigas epigéicas de fragmentos de Mata Atlântica situados em regiões altamente urbanizadas. Foram determinadas a riqueza e a frequiência de ocorrência das espécies, e também a similaridade entre os fragmentos.

\section{MATERIAL E MÉTODOS}

A área de estudo é formada por três fragmentos de Mata Atlântica, de composição florística secundária, localizada na cidade de São Paulo. O Parque da

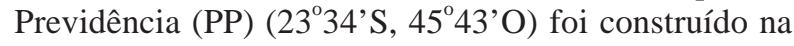
década de 80 e as famílias Anacardiaceae, Fabaceae, Cecropiaceae e Euphorbiaceae (QUERIDO, 1999) estão aí muito bem representadas. A área de 9,1 ha sofreu alguns incêndios ao longo de sua existência e apenas uma pequena parte do Parque ainda recebe visitação pública. As armadilhas foram colocadas em um local onde não ocorre visitação. O Horto Oswaldo Cruz (HOC) (23 33 'S, $46^{\circ} 43^{\circ} \mathrm{O}$ ) possui 2,0 ha, até o início do século 20 pertencia à fazenda Butantan, que era praticamente coberta por pastagens. Atualmente o local se encontra fechado para o público e, nas áreas mais preservadas, onde foram colocadas as armadilhas, há o predomínio de duas espécies de palmeiras: Roystonea oleracea e Archontophoenix cunninghamiana. A Reserva Florestal Armando Salles de Oliveira (CUASO) $\left(23^{\circ} 33^{\prime} \mathrm{S}, 46^{\circ} 43^{\prime} \mathrm{O}\right)$ possui 10 ha, também fazia parte da fazenda Butantan e possui uma vegetação rica, com predominância das famílias Euphorbiaceae, Meliaceae, Fabaceae, Moraceae e Arecaceae. Atualmente, esse fragmento é apenas destinado à pesquisa, pertencendo à Universidade de São Paulo (USP).

Foram realizadas quatro coletas em cada fragmento nos meses de março, junho, setembro e dezembro de 2001. Delimitaram-se cinco fileiras (amostras 1, 2, 3, 4 e 5) distando 10 metros entre si, sendo que em cada uma foram colocados dez frascos de "pitfall", com um metro de espaçamento entre cada um. Os frascos (copos de plástico de $500 \mathrm{ml}$ e $14 \mathrm{~cm}$ de diâmetro) continham álcool 70\%, permanecendo em cada fragmento por uma semana.

$\mathrm{O}$ material inicialmente foi identificado em subfamílias (Bolton, 2003) e em gêneros (BoLton, 1994). A separação em morfoespécies baseou-se na coleção de referência das formigas do Alto Tietê (Universidade de Mogi das Cruzes/SP), onde estão depositados os exemplares estudados.

Para a análise dos dados, as quatro coletas foram agrupadas sem separação temporal das amostras de cada fragmento. O material biológico coletado nos "pitfall" foi acrescentado no primeiro "pitfall" da fileira. Os resultados foram tabulados (colunas = amostras; linhas = espécies) baseando-se em dados de presença e ausência das espécies, pois segundo RoMERo \& JAFFé (1989), para os insetos sociais apenas um indivíduo forrageando é um indicador da existência da colônia. Assim, com esses dados foram calculadas a frequiência de ocorrência de cada espécie e a estimativa de riqueza (Chao 2), usando a versão 7.5 do programa EstimateS (Colwell, 2005). A partir da matriz de presença e ausência das espécies foi calculado o índice de similaridade de Jaccard entre as amostras e elaborado o dendograma utilizando-se o algoritmo de agrupamento pela média (MAGURRAN, 1988; KREBS, 1989). As amostras foram ordenadas através de um NMDS (Non-Metric Multidimensional Scaling); utilizou-se a distância de Bray-Curtis para se medir a similaridade entre as amostras (CLARKE, 1993). A diferença entre as três assembléias de formigas (PP, CUASO, HOC) foi testada por uma análise de similaridade, ANOSIM, que verifica a significância de um valor $\mathrm{R}$ que mede a associação entre elas (CLARKE, 1993).

\section{RESULTADOS}

Em todos os fragmentos estudados foram coletadas 79 espécies de formigas (17.435 espécimes), pertencentes a nove subfamílias e 32 gêneros. No PP foram registradas 62 espécies, na CUASO 46 e no HOC 43 (Tab. I), sendo estimadas 14,10 e 32 espécies a mais para cada localidade, respectivamente (Fig. 1).

As subfamílias mais ricas foram Myrmicinae com 49\% das espécies, seguida por Ponerinae $19 \%$, Formicinae $12 \%$, Dolichoderinae 5\%, Heteroponerinae e Pseudomyrmecinae 4\%, Ectatominae e Ecitoninae com 2,5\% e Proceratiinae com 1,5\%. Os gêneros Pheidole e Hypoponera apresentaram o maior número de espécies, 11 e nove respectivamente (Tab. I). A freqüência de ocorrência variou entre as espécies e entre os fragmentos (Tab. I). Entretanto espécies como Cyphomyrmex major (Mayr, 1862), Cyphomyrmex sp. 2, Pheidole sp. 3, Pheidole sp. 7, Solenopsis (Diplorhoptrum) sp. 1, Solenopsis sp. 4, Brachymyrmex sp. 1, Camponotus sp. 1, Paratrechina fulva Mayr, 1862, Gnamptogenys striatula (Mayr, 1864), Ectatomma edentatum Roger, 1863, Pachycondyla constricta Mayr, 1884, P. striata Smith, 1858 e Linepithema humile Mayr, 1868 apresentaram alta freqüência de ocorrência em pelo menos dois fragmentos (Tab. I). No geral, a maioria dos gêneros coletados pode ser caracterizada como generalista, exceto Acanthognathus, Oxyepoecus, Pyramica, Gnamptogenys, Heteroponera, Discothyrea e as espécies de Attini, que possuem hábitos alimentares mais especializados.

Em todos os fragmentos verificou-se a presença de gêneros normalmente encontrados nos ecossistemas urbanos, como Pheidole, Solenopsis, Brachymyrmex, Camponotus, Paratrechina, Tapinoma e Linepithema. Porém, ao mesmo tempo, foram observadas porcentagens relativamente altas de espécies pertencentes às subfamílias de Poneromorfas (sensu Bolton, 2003), ou seja, $19 \%$ no PP, $24 \%$ na CUASO e $15 \%$ no HOC. Comparando-se as três localidades nota-se que $29 \%$ das espécies são compartilhadas entre elas; $40 \%$ entre PP e CUASO; $38 \%$ entre PP e HOC e $35 \%$ entre CUASO e HOC. Em relação às espécies exclusivas, foram constatadas $21 \%$ no PP, $10 \%$ na CUASO e $4 \%$ no HOC, 

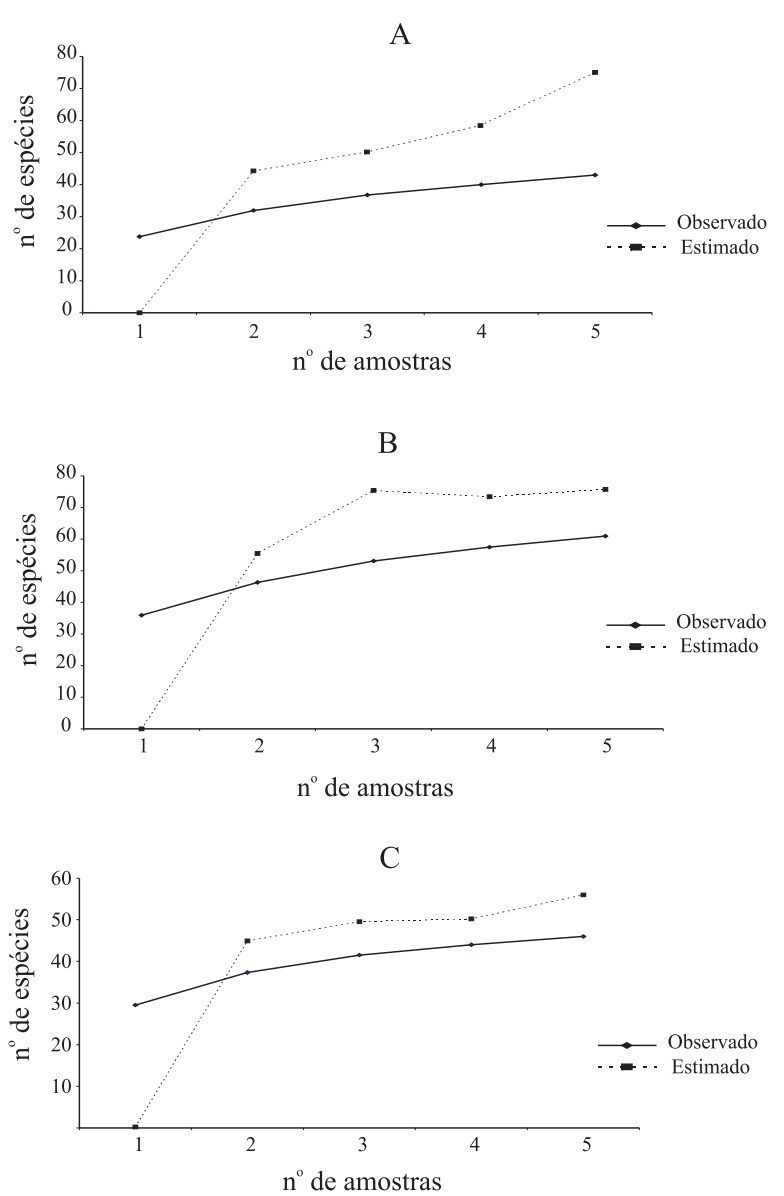

Fig 1. Riqueza observada e estimada (Chao2) de acordo com a área estudada, São Paulo, SP (A, Horto Oswaldo Cruz; B, Parque da Previdência; C, Reserva Florestal Armando Salles de Oliveira). enquanto de formigas andarilhas $6,5 \%, 4 \%$ e $2,5 \%$, respectivamente.

O dendograma dos índices de similaridade de Jaccard entre as amostras (Fig. 2), mostra que as amostras são mais similares entre si dentro de uma mesma área de estudo, com exceção da amostra 2 de HOC, que foi diferente das demais. Analisando-se as áreas de estudo, verifica-se que PP e CUASO são mais similares entre si, enquanto que $\mathrm{HOC}$ foi ligeiramente mais dissimilar em relação às duas anteriores.

Na ordenação das amostras através do NMDS (Fig. 3 ), verifica-se que as assembléias de formigas das três áreas de estudo diferiram entre si, formando três grupos distintos: HOC na parte superior do gráfico; CUASO à esquerda; e PP abaixo. $O$ valor de $\mathrm{R}_{\mathrm{ANOSIM}}=0,844$ foi significativo $(\mathrm{p}<0,001)$, confirmando que as assembléias foram distintas entre as áreas.

\section{DISCUSSÃO}

A riqueza de espécies amostrada é $21 \%$ maior que a do trabalho realizado por Feitosa \& Ribeiro (2005) no Parque Estadual da Cantareira (PEC), também situado na cidade de São Paulo e cuja área de Mata Atlântica representa o maior maciço florestal urbano do mundo. Entretanto, em ambos trabalhos as curvas de acumulação de espécies não se estabilizaram; segundo Longino et al. (2002) e LEPONCE et al. (2004), em áreas tropicais esse tipo de resultado se deve ao alto número de espécies raras nas amostras.

Em relação à composição da fauna, nota-se que apenas 18 gêneros foram compartilhados pelas áreas urbanizadas estudadas e o PEC. Dentre as espécies identificadas, tem-se Octostruma rugifera, $P$. denticulata,

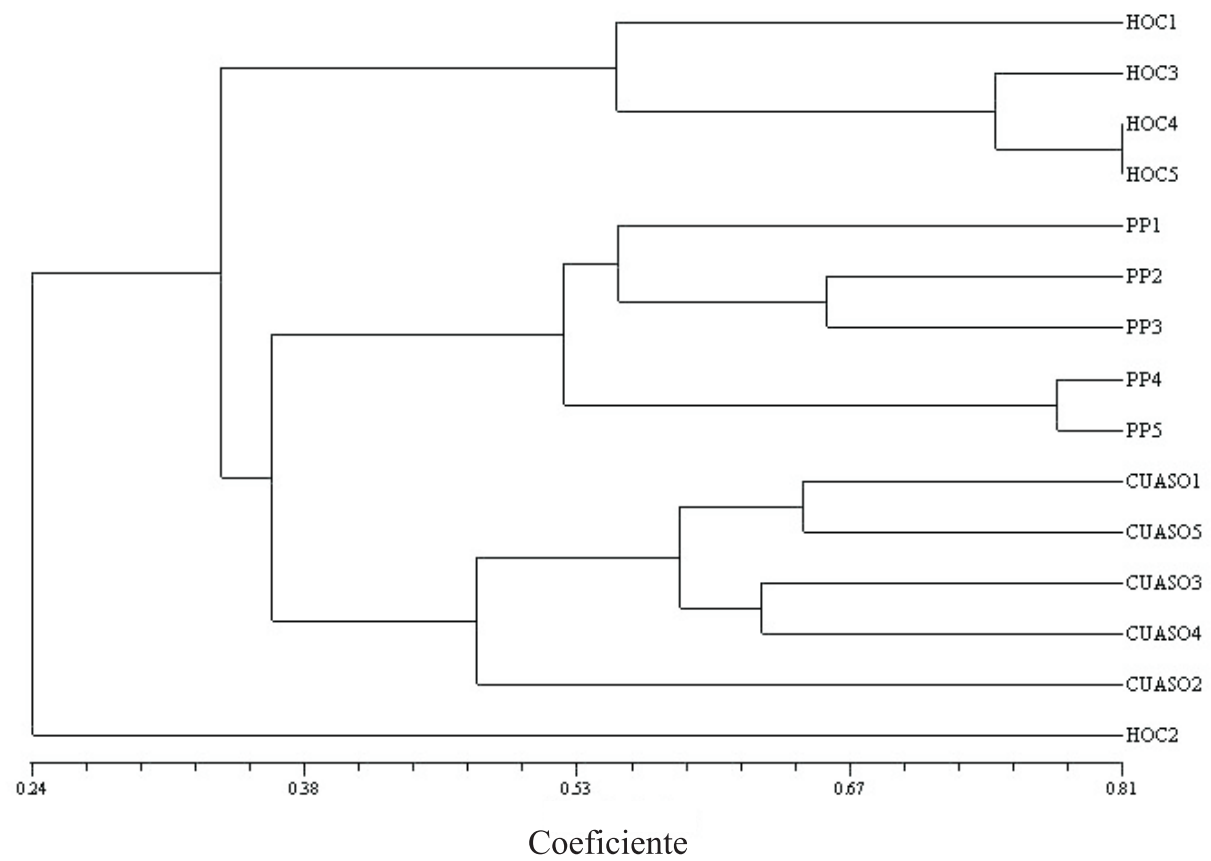

Fig. 2. Similaridade (Índice de Jaccard) entre as amostras da fauna de formigas nos fragmentos de Mata Atlântica estudados na cidade de São Paulo (HOC, Horto Oswaldo Cruz; PP, Parque da Previdência; CUASO, Reserva Florestal Armando Salles de Oliveira). 
Tabela I. Freqüência relativa (número total de aparecimentos) de cada espécie de formiga amostrada de acordo com os fragmentos estudados (PP, Parque da Previdência; CUASO, Reserva Florestal Armando Salles de Oliveira; HOC, Horto Oswaldo Cruz).

\begin{tabular}{|c|c|c|c|}
\hline Subfamílias/espécies & $\mathrm{PP}$ & CUASO & $\mathrm{HOC}$ \\
\hline \multicolumn{4}{|l|}{ MYRMICINAE } \\
\hline Acanthognatus rudis Brown \& Kempf, 1969 & $1,11(2)$ & $0,70(1)$ & \multirow{4}{*}{$0,84(1)$} \\
\hline \multicolumn{3}{|l|}{ Acromyrmex niger (Smith,1858) } & \\
\hline Apterostigma sp. 1 & $2,77(5)$ & \multirow[t]{7}{*}{$0,70(1)$} & \\
\hline Cephalotes sp. 1 & $0,55(1)$ & & \\
\hline Crematogaster sp. 1 & $1,67(3)$ & & $2,52(3)$ \\
\hline Crematogaster sp. 2 & $0,55(1)$ & & $0,84(1)$ \\
\hline Crematogaster sp. 3 & $2,22(4)$ & & $0,84(1)$ \\
\hline Crematogaster sp. 4 & $0,55(1)$ & & \\
\hline Mycetosoritis sp. 1 & $0,55(1)$ & & \\
\hline Cyphomyrmex major (Mayr, 1862) & $2,77(5)$ & $2,80(4)$ & \\
\hline Cyphomyrmex sp. 1 (gr. strigatus) & & $2,10(3)$ & \\
\hline Cyphomyrmex sp. 2 (gr. strigatus) & $2,22(4)$ & $2,80(4)$ & $3,36(4)$ \\
\hline Myrmicocrypta sp. 1 & & $3,50(5)$ & \\
\hline Octostruma rugifera (Mayr, 1887) & $0,55(1)$ & & \\
\hline Oxyepoecus rastratus (Mayr, 1887) & $0,55(1)$ & & $0,84(1)$ \\
\hline Oxyepoecus sp. 1 & $1,11(2)$ & & $4,20(5)$ \\
\hline Pheidole megacephala (Fabricius, 1793) & $0,55(1)$ & & $0,84(1)$ \\
\hline Pheidole sp. 1 & $1,67(3)$ & & \\
\hline Pheidole sp. 2 & $0,55(1)$ & & \\
\hline Pheidole sp. 3 & $2,77(5)$ & $2,80(4)$ & $3,36(4)$ \\
\hline Pheidole sp. 4 & $1,67(3)$ & $1,40(2)$ & $1,68(2)$ \\
\hline Pheidole sp. 5 & $2,22(4)$ & & \\
\hline Pheidole sp. 6 & & $3,50(5)$ & \\
\hline Pheidole sp. 7 & $2,77(5)$ & $2,10(3)$ & $3,36(4)$ \\
\hline Pheidole sp. 8 & & & $1,68(2)$ \\
\hline Pheidole sp. 9 & & $0,70(1)$ & $2,52(3)$ \\
\hline Pheidole sp. 10 & $0,55(1)$ & & \\
\hline Pyramica denticulata Mayr, 1887 & $1,67(3)$ & $2,10(3)$ & $2,52(3)$ \\
\hline P. elongata Emery & $0,55(1)$ & & $0,84(1)$ \\
\hline P. crassicornis Emery & $2,77(5)$ & & $0,84(1)$ \\
\hline Pyramica sp. 1 & $0,55(1)$ & & \\
\hline Strumigenys louisianae Roger, 1863 & $1,11(2)$ & & \\
\hline Solenopsis (Diplorhoptrum) sp. 1 & $2,77(5)$ & $3,50(5)$ & $1,68(2)$ \\
\hline Solenopsis sp. 1 & $1,67(3)$ & $0,70(1)$ & $4,20(5)$ \\
\hline Solenopsis sp. 2 & $1,11(2)$ & $2,10(3)$ & $4,20(5)$ \\
\hline Solenopsis sp. 3 & $0,55(1)$ & & $3,36(4)$ \\
\hline Solenopsis sp. 4 & $2,77(5)$ & $3,50(5)$ & $2,52(3)$ \\
\hline Wasmannia auropunctata Roger, 1863 & $0,55(1)$ & & \\
\hline Wasmannia sp. 1 & $2,77(5)$ & $1,40(2)$ & \\
\hline FORMICINAE & & & \\
\hline Brachymyrmex sp. 1 & $2,77(5)$ & $2,10(3)$ & $3,36(4)$ \\
\hline B. patagonicus (Mayr, 1868) & $0,55(1)$ & & \\
\hline Camponotus crassus Mayr, 1862 & $0,55(1)$ & & \\
\hline Camponotus sp. 1 & $2,77(5)$ & $2,10(3)$ & $4,20(5)$ \\
\hline Camponotus sp. 2 & $1,67(3)$ & & $2,52(3)$ \\
\hline Myrmelachista sp. 1 & $1,67(3)$ & $3,50(5)$ & $0,84(1)$ \\
\hline Mymelachista sp. 2 & & $0,70(1)$ & \\
\hline Paratrechina fulva Mayr, 1862 & $2,77(5)$ & $3,50(5)$ & $3,36(4)$ \\
\hline P. longicornis (Latreille, 1802) & & $2,10(3)$ & \\
\hline Paratrechina sp. 1 & $0,55(1)$ & & \\
\hline ECTATOMMINAE & & & \\
\hline Gnamptogenys striatula (Mayr, 1884) & $2,77(5)$ & $3,50(5)$ & $4,20(5)$ \\
\hline Ectatomma edentatum Roger, 1863 & $2,77(5)$ & $0,70(1)$ & $3,36(4)$ \\
\hline HETEROPONERINAE & & & \\
\hline Heteroponera mayri (Kempf, 1962) & $2,22(4)$ & $2,10(3)$ & \\
\hline H. dentinodis (Mayr, 1887) & & $0,70(1)$ & \\
\hline H. dolo (Roger, 1860) & $0,55(1)$ & $2,10(3)$ & \\
\hline PROCERATIINAE & & & \\
\hline Discothyrea sexarticulata (Borgmeier, 1954) & $0,55(1)$ & 0,70 & \\
\hline PONERINAE & & & \\
\hline Hypoponera sp. 1 & $2,77(5)$ & & $0,84(1)$ \\
\hline Hypoponera sp. 2 & $2,77(5)$ & & \\
\hline Hypoponera sp. 3 & $1,11(2)$ & $3,50(5)$ & $0,84(1)$ \\
\hline Hypoponera sp. 4 & $1,11(2)$ & $2,80(4)$ & $4,20(5)$ \\
\hline Hypoponera sp. 5 & & $2,80(4)$ & $0,84(1)$ \\
\hline Hypoponera sp. 6 & & $2,10(2)$ & $0,84(1)$ \\
\hline Hypoponera sp. 7 & & $2,10(2)$ & \\
\hline Hypoponera sp. 8 & $2,77(5)$ & $2,80(4)$ & \\
\hline Hypoponera sp. 9 & & $2,10(3)$ & \\
\hline Leptogenys sp. 1 & $1,67(3)$ & $2,10(3)$ & $0,84(1)$ \\
\hline
\end{tabular}


Tabela I (cont.)

\begin{tabular}{|c|c|c|c|}
\hline Subfamílias/espécies & PP & CUASO & $\mathrm{HOC}$ \\
\hline Odontomachus affinis (Guérin-ménenville, 1844) & $1,11(2)$ & $3,50(5)$ & $2,52(3)$ \\
\hline O. meinerti (Forel, 1905) & & $0,70(1)$ & $4,20(5)$ \\
\hline O. chelifer (Latreille, 1802) & $2,77(5)$ & $2,10(2)$ & \\
\hline Pachycondyla constricta (Mayr, 1884) & $2,77(5)$ & $3,50(5)$ & $1,68(2)$ \\
\hline P. striata (Smith, 1858) & $2,77(5)$ & $3,50(5)$ & $3,36(4)$ \\
\hline \multicolumn{4}{|l|}{ DOLICHODERINAE } \\
\hline Azteca sp. 2 & $0,55(1)$ & & \\
\hline Tapinoma melanocephalum (Fabricius, 1793) & $1,11(2)$ & & \\
\hline Linepithema humile (Mayr, 1868) & $2,77(5)$ & $3,50(5)$ & $0,84(1)$ \\
\hline Linepithema sp. 1 & $1,11(2)$ & $2,10(3)$ & \\
\hline \multicolumn{4}{|l|}{ ECITONINAE } \\
\hline Labidus praedator (F. Smith, 1858) & $1,11(2)$ & & $0,84(1)$ \\
\hline L. coecus (Latreille, 1802) & & $2,10(3)$ & $4,20(5)$ \\
\hline \multicolumn{4}{|l|}{ PSEUDOMYRMECINAE } \\
\hline Pseudomyrmex gracilis (Fabricius, 1804) & & & $1,68(2)$ \\
\hline P. oculatus (Smith, 1855) & $1,11(2)$ & & \\
\hline P. pallidus (F. Smith, 1858) & & $0,70(1)$ & $0,84(1)$ \\
\hline TOTAL DE ESPÉCIES & 62 & 46 & 43 \\
\hline
\end{tabular}

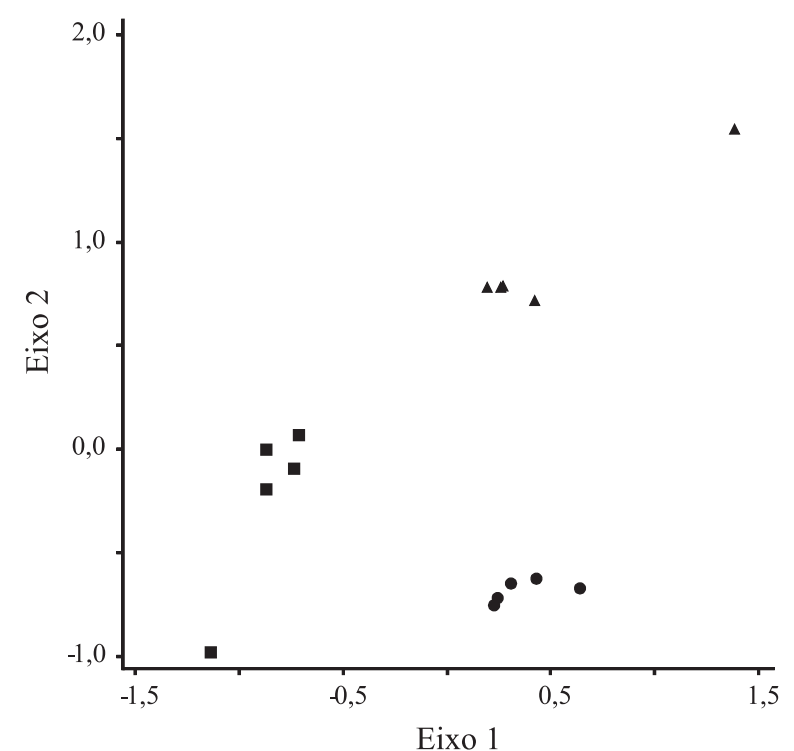

Fig. 3. Ordenação das amostras de formigas dos fragmentos estudados, através de um NMDS (Non-Metric Multidimensional Scaling). ( $\boldsymbol{\Delta}$, Horto Oswaldo Cruz; @, Parque da Previdência; $\square$, Reserva Florestal Armando Salles de Oliveira).

G. striatula, H. mayri, $H$. dolo, D. sexarticulata e $P$. striata. Deve-se ressaltar, porém, que os métodos de coleta e a época de amostragem foram diferentes em ambos os trabalhos.

A subfamília Myrmicinae foi amostrada com o maior número de táxons, corroborando WARD (2000) e DELABIE et al. (2000a) para a fauna de formigas de serapilheira em áreas de Mata Atlântica e também SiLVA \& SiLVESTRE (2004) para a fauna de formigas de solo. Segundo Fowler et al. (1991), os Myrmicinae constituem um dos grupos de formigas mais diversificados em relação aos hábitos de alimentação e de nidificação. Outras subfamílias que se destacaram foram aquelas que pertencem aos atuais poneromorfos, normalmente táxons com comportamento de alimentação diferenciado; a subfamília Ponerinae, por exemplo, foi representada por 15 espécies.

Dentre os Ponerinae foram coletados exemplares de Odontomachus que (apesar de suas espécies conspícuas e comuns no solo (LATTKe, 2003)) não foi amostrado por FeItosa \& Ribeiro (2005) no PEC; (mas, Frei Walter Kempf, nas décadas de 1950 e 1960, obteve exemplares nesta área) (Feitosa \& Ribeiro, 2005). Indivíduos deste gênero são predadores agressivos (LATTKE, 2003) e são sensíveis às mudanças ambientais (Delabie et al., 2000b). Seu padrão de ocorrência, com três espécies na CUASO, mostra que as condições abióticas e bióticas para a sua manutenção ainda estão adequadas (apesar do fragmento estar situado em um ecossistema altamente urbanizado).

Pheidole e Hypoponera foram os gêneros encontrados com o maior número de espécies, porém, são de ampla ocorrência na serapilheira da Região Neotropical (WARD, 2000). Em relação à alimentação, WEISER \& KASPARI (2006) os classificaram como generalistas e predadores hipogéicos de serapilheira, respectivamente. Porém, no geral nota-se que as espécies/morfoespécies de maior ocorrência nos fragmentos estudados são generalistas hipogéicas ou epigéicas, o que provavelmente reflete as influências da malha urbana do entorno.

Por outro lado, verifica-se a presença de espécies que possuem comportamentos diferenciados como $H$. mayri, H. dolo e H. dentinodis, gênero que possui hábitos de nidificar em troncos e galhos caídos (LATTKE, 2003), cuja alimentação ainda é desconhecida (WEISER \& KASPARI, 2006); indivíduos de Discothyrea são predadores de ovos de artrópodes como aranhas e Chilopoda (Delabie et al., 2000a); as espécies de Pyramica são especializadas em predar Collembola (DEJEAn, 1987), ou então gêneros especialistas como Acanthognathus, Gnamptogenys, Oxyepoecus e os pertencentes à Tribo Attini. O fato de Myrmelachista sp.1 ter sido amostrada em três fragmentos, sendo que em dois deles com alta freqüência de ocorrência, merece destaque, pois representantes deste gênero possuem biologia e alimentação desconhecidas (WEISER \& KASPARI, 2006); os trabalhos que se tem na literatura os associa à vegetação (FERNÁNDEZ, 2003).

No geral, a composição da fauna de formigas é bem diferenciada entre os três fragmentos estudados, como mostra a análise de ordenação. Das 79 espécies encontradas, somente 22 são compartilhadas e, dentre essas, se destacam O. affinis, G. striatula e P. striata, de hábitos especializados. 
Analisando-se os fragmentos, observa-se que PP e CUASO são mais similares entre si, mesmo que o primeiro tenha sofrido alguns incêndios ao longo de sua existência e tenha um histórico de preservação recente. Assim, os dois fragmentos parecem possuir nichos de alimentação e de nidificação semelhantes, o que pode estar relacionado ao tamanho das duas áreas. Segundo HÖLLDOBLER \& WilsON (1990) e RosENZWEIG (1995), áreas maiores podem favorecer maior oferta de nichos disponíveis e de recursos e, consequentemente, haverá maior número de espécies (YAMAGUCHI, 2004).

A dissimilaridade entre HOC e CUASO reforça esse resultado, pois são áreas diferentes em tamanho (a primeira é 4,5 vezes maior do que a segunda), porém ambos pertenciam à fazenda Butantan, cujo cultivo principal era de Poaceae para a formação de pastagens. Assim, mesmo durante o processo de regeneração da vegetação de ambos os fragmentos, que deve ter sido semelhante devido ao histórico dos locais, a composição da mirmecofauna diferenciou-se.

Analisando-se a composição da fauna de formigas denominadas de andarilhas - características de áreas domiciliares e peri-domiciliares - é possível observar que PP apresenta cinco espécies (Ph. megacephala; W. auropunctata; P. fulva; T. melanocephalum e L. humile) das sete espécies consideradas por CAMPOS-FARINHA et al. (2002) como de importância nas áreas urbanas do Brasil; na CUASO três espécies desta formiga foram registradas ( $P$. fulva, $P$. longicornis e $L$. humile) e no HOC apenas duas (P. fulva e L. humile). Esses táxons compartilham características que facilitam sua introdução a novos ambientes, como dieta generalista, poliginia, redução da agressividade intra-específica, pequeno tamanho e recrutamento em massa, taxa de migração elevada, população unicolonial, abolição do vôo nupcial, além de operárias e rainhas muito pequenas (PASSERA, 1994; HoLWAY et al., 2002).

Em conseqüência dos aspectos biológicos apresentados, as formigas andarilhas podem deslocar ou até mesmo extinguir alguns táxons dos fragmentos. É o caso de $L$. humile, que foi encontrada nos três locais em dois deles com alta frequiência de ocorrência - pois é uma espécie característica de ambientes desestruturados (VEGA \& RUST, 2001), além de interferir com a diversidade das áreas que invadem (Schultz \& McGlynn, 2000). Dessa forma, essas espécies podem causar uma diminuição ainda maior da diversidade dos fragmentos de Mata Atlântica da cidade de São Paulo devido à perda das interações biológicas existentes.

Agradecimentos. À Fundação de Amparo ao Ensino e Pesquisa (FAEP/UMC) pelo auxílio financeiro, ao Dr. Rogério R. Silva (MZUSP) pela confirmação na identificação das espécies e ao Dr. Antonio D. Brescovit (Instituto Butantan) pelo material biológico concedido.

\section{REFERÊNCIAS BIBLIOGRÁFICAS}

BLAIR, R. B. 1996. Land use and avian species diversity along an urban gradient. Ecological Applications 6:506-519.

Bolton, B. 1994. Identification guide to the ant genera of the world. Cambridge, Harvard University. 222p.

2003. Synopsis and classification of Formicidae. Memoirs of the American Museum Entomological Institute 71:1-370.
Bueno, O. C. \& Campos-Farinha, A. E. C. 1999. As formigas domésticas. In: MARICONI, F. A. M. coord. Insetos e outros invasores de residências. Piracicaba, FEALQ, pp. 135-180.

Campos-Farinha, A. E. C.; Bueno, O. C. \& Kato, L. M. 2002. As formigas urbanas no Brasil: retrospecto. Biológico 64:129-133.

Clarke, K. R. 1993. Non-parametric multivariate analysis of changes in community structure. Australian Journal of Ecology 18:117-143.

Colwell, R. K. 2005. EstimateS: Statistical estimation of species richness and shared species from samples. Version 7.5 User's guide and application. Disponível em: <http:// viceroy.ceb.uconn.edu/EstimateS>. Acesso em: 02.05.2005.

Connor, E. F.; Hafernik, J.; Moore, V. L. \& Rickman, J. K. 2002. Insect conservation in an urban biodiversity hotspot: the San Francisco Bay area. Journal of Insect Conservation 6:247-259.

Crooks, K. R. \& Soulé, M. E. 1999. Mesopredator release and avifaunal extinctions in a fragmented system. Nature 400:563-566.

Dejean, A. 1987. Behavioral plasticity of hunting works of Serrastruma serrula presented with different arthropods. Sociobiology 13:191-208.

Delabie, J. H. C., Agosti, D. \& Nascimento, I. C. 2000a. Litter ant communities of the Brazilian Atlantic rain forest region. In: Agosti, D.; Majer, J.; Alonso, L. \& Schultz, T. eds. Sampling ground-dwelling ants: case studies from the worlds rain forests. Cap. 1. School of environmental biology, Bulletin n. 18, p. 1-10.

Delabie, J. H. C.; Nascimento, I. C. \& Mariano, C. S. F. 2000b. Importance de l'agriculture cacaoy'ere pour le maintien de la biodiversité: etude comparée de la myrmécofaune de différents milieux du sud-est de Bahia, Brésil (Hymenoptera, Formicidae). In: International Cocoa Research Conference, XII, Salvador, Anais... Salvador, Brasil. p.41.

Delabie, J. H. C.; Nascimento, I. C. do; Pacheco, P. \& Casimiro, A B. 1995. Community structure of house-infesting ants (Hymenoptera: Formicidae). Florida Entomologist 78:264-270.

Feitosa, R. S. M. \& Ribeiro, A. S. 2005. Mirmecofauna (Hymenoptera: Formicidae) de serapilheira de uma área de Floresta Atlântica no Parque Estadual da Cantareira - São Paulo, Brasil. Biotemas 18:51-71.

Fernández, F. 2003. Subfamília Formicinae. In: Fernández, F. ed. Introduccion a las hormigas de la región Neotropical Bogotá, Instituto de Investigación de Recursos Biológicos Alexander Von Humbolt. p. 299-306.

Fowler, H. G.; Forti, L. C.; Brandão, C. R. F.; Delabie, J. H. C. \& Vasconcelos, H. L. 1991. Ecologia nutricional de formigas. In Panizzi, A. R.; Parra, J. R. P. eds. Ecologia nutricional de insetos. São Paulo, Manole. p.131-223.

Hölldobler, B. \& Wilson, E. O. 1990. The ants. Cambridge: Belknap. 732p.

Holway, D. A.; Lach, L.; Suarez, A. V.; Tsutsui, N. D. \& Case, T. J. 2002. The causes and consequences of ant invasion. Annual of Review of Ecology and Systematics 33:181-233.

KREBS, C. J. 1989. Ecological methodology. New York, Harper Collins. 645p.

LattKe, J. E. 2003. Biogeografia de las hormigas neotropicais. In FERNÁNDEZ, F. ed. Introduccion a las hormigas de la región Neotropical. Bogotá, Instituto de Investigación de Recursos Biológicos Alexander Von Humbolt. p. 65-85.

Leponce, M.; Theunis, L.; Delabie, J. H. C. \& Roisin, Y. 2004. Scale dependence of diversity measures in a leaf-litter ant assemblages. Ecography 27:253-267.

Longino, J. T.; Coddington, J. \& Colwell, R. K. 2002. The ant fauna of tropical rain forest: estimating species richness three different ways. Ecology 83:689-702.

López-Moreno, I. R. \& Diaz-Betancourt, M. E. 1995. El Studio de la biodiversidad en ecosistemas urbanos. Arbor 596:63-86.

López-Moreno, I. R.; Diaz-Betancourt, M. E.; Landa, T. S. 2003. Insectos sociales em ambientes antropizados: las hormigas de la ciudad de Coatepec, Veracruz, México. Sociobiology 42:605-622

Magurran, A. E. 1988. Ecological diversity and its measurement. Princeton, Princeton University. 167p.

McInTYRe, N. E. 2000. Ecology of urban arthropods: a review and a call to action. Annals of the Entomological Society of 
America 93:825-835.

McIntyre, N. E.; RAngo, J.; FAGAN, W. F. \& FaEth, S. H. 2001. Ground arthropod community structure in a heterogeneous urban environment. Landscape and urban planning 52:257-274.

Murphy, D. D. 1997. Desafios à diversidade biológica em áreas urbanas. In: Wilson, E. O. \& Peter, F. M. eds. Biodiversidade. Rio de Janeiro, Nova Fronteira. p.89-97.

Passera, L. 1994. Characteristcs of tramp species. In: Williams, D. F. ed. Exotic ants: biology, impact and control of introduced species. Boulder, Westview. pp. 23-43.

Querido, M. J. S. 1999. Butantan e suas veredas, guia cultural e turístico. São Paulo, Perez. 30p.

Rodrigues, J. J. S.; Brown JR., K. S. \& RuszczyK, A. 1993. Resources and conservation of neotropical butterflies in an urban forest fragments. Biological Conservation 64:3-9.

Romero, H. \& JAFFÉ, K. 1989. A comparison of methods for sampling ants (Hymenoptera: Formicidae) in savannas. Biotropica 21:348-352.

Rosenzweig, M. L. 1995. Species diversity in space and time. Cambridge, Cambridge University. 346p.

Schultz, T. R. \& McGlynn, T. 2000. The interactions of ants with other organism. In: Agosti, D.; MaJer, J. D.; Alonso, L. E.
\& Schultz, T. R. eds. Ants: Standard methods for measuring and monitoring biodiversity. Smithsonian Institution. p. 35-44

Silva, R. R. \& Silvestre, R. 2004. Riqueza da fauna de formigas (Hymenoptera: Formicidae) que habita as camadas superficiais do solo em Seara, Santa Catarina. Papéis Avulsos de Zoologia 44:1-11.

Suarez, A. V.; Bolger, D. T.; Case, T. J. 1998. Effects of fragmentation and invasion on native ant communities in coastal southern California. Ecology 79:2041-2056.

VegA, S. J. \& Rust, M. K. 2001. The Argentine ant: a significant invasive species in agricultural, urban and natural environments. Sociobiology 37:3-25.

WARD, P. S. 2000. Broad-scale patterns of diversity in leaf litter ant communities. In: Agosti, D.; Majer, J. D.; Alonso, L. E. \& Schultz, T. eds. Ants: Standard methods for measuring and monitoring biodiversity. Smithsonian Institution. p.99-121.

Weiser, M. \& Kaspari, M. 2006. Ecological morphospace of New World ants. Ecological Entomology 31:131-142.

YAMAGUCHI, T. 2004. Influence of urbanization on the distribution in parks of Tokyo and Chiba City, Japan. I. Analysis of ant species richness. Ecological Research 19:209-216. 